\title{
Narrowband Control Design for Smart Structural Systems
}

\author{
Daniel J. Kolepp and Ralph C. Smith ${ }^{1}$ \\ Center for Research in Scientific Computation \\ Department of Mathematics \\ North Carolina State University \\ Raleigh, NC 27695-8205
}

\begin{abstract}
This investigation focuses on the development of narrowband control strategies which are effective for structural systems subjected to both harmonic exogenous forces and stochastic uncertainties in the model or measurements. A cantilever beam with surface-mounted piezoceramic actuators is used as a prototypical structure since it exhibits physical attributes of a variety of vibrating structures but is sufficiently simple to facilitate initial control design. A PDE model for the structure is discretized using a spline-based Galerkin technique to obtain a semi-discrete system that is appropriate for finite-dimensional control design. A narrowband control method, which can be tuned to attenuate either natural frequencies or specific frequencies in the exogenous input, is developed and compared with standard LQR techniques through numerical examples.
\end{abstract}

\footnotetext{
${ }^{1}$ Corresponding Author: rsmith@eos.ncsu.edu, (919) 515-7552
} 


\section{Introduction}

Piezoceramic elements have proven highly successful in a wide range of structural, structural acoustic, and fluid-structure systems due to their dual actuator and sensor capabilities and their broadband response. These attributes are augmented by the fact that piezoceramic patches are lightweight, and hence do not significantly alter the passive dynamics of the underlying structure, and are relatively inexpensive to fabricate. In combination, these properties can be utilized to design controllers which are effective for a large range of both stochastic and harmonic structural dynamics. Furthermore, by utilizing inherent coupling between structural vibrations and adjacent fields, PZT transducers can be employed for structural acoustic control design or control of adjacent flows.

In this paper, we focus on the development of narrowband control designs for structural systems which exhibit strong harmonic responses or are driven by periodic exogenous forces. Such exogenous forces include engine noise, electromagnetic inputs, or disturbances due to rotating components. The goal of attenuating harmonic structural responses has been augmented by the development of composites materials which are lightweight and provide minimal damping. Finally, all systems are subject to some degree of stochastic uncertainty due to either broadband or stochastic exogenous forces (e.g., turbulence) or limitations in the models, numerical approximations, or control hardware. Hence the narrowband control designs will ultimately be combined with feedback mechanisms to provide sufficient robustness for a range of applications.

To provide a regime which facilitates model development, analysis, and numerical approximation while retaining attributes of a range of structural systems, we consider the design of narrowband control techniques in the context of a cantilever beam with surface-mounted piezoceramic patches. This illustrates a number of the associated modeling and numerical issues and illustrates the capabilities of the control techniques for a prototypical system. These techniques can then be extended to more complex physical systems once the systems are quantified by finite dimensional models (e.g., finite element or modal).

Appropriate models and numerical techniques will be developed in Section 2 and control designs comprised of narrowband and feedback components will be discussed in Section 3. A brief summary of existing control methods for smart structural systems will also be provided in that discussion. Numerical examples illustrating the control techniques will be provided in Section 4.

\section{Model and Numerical Approximation Techniques}

We illustrate the development of narrowband control techniques in the context of a cantilever beam with surface-mounted piezoceramic patches as depicted in Figure 1. For modeling purposes, the beam is assumed to have length $\ell$, thickness $h_{b}$, and width $b$, and is oriented along the $x$-axis with
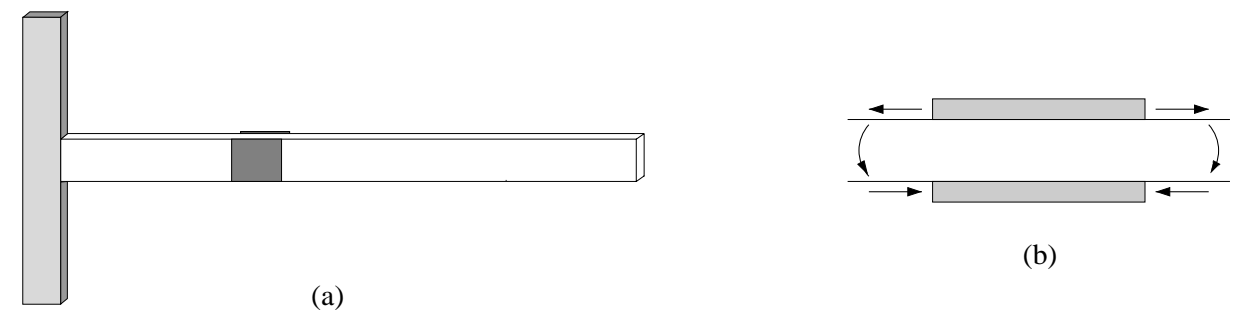

(b)

Figure 1. (a) Thin beam with surface-mounted piezoceramic patches. (b) Bending moments generated by diametrically out-of-phase voltages to the patches. 
transverse vibrations $w$ occurring in the $z$-direction. The density, Young's modulus, and KelvinVoigt damping coefficients for the beam are respectively denoted by $\rho_{b}, E_{b}$ and $c_{D}$ and analogous quantifies for the PZT patch are denoted by $\rho_{p z t}, E_{p z t}, c_{p z t}$. The coefficient for air (viscous) damping is denoted by $\gamma$ and exogenous forces are represented by $g$. Finally, the patches are assumed to have thickness $h_{p z t}$ and cover the region $p_{1} \leq x \leq p_{2}$.

As detailed in [3], force and moment balancing yield the PDE model

$$
\begin{aligned}
& \rho(x) \frac{\partial^{2} w}{\partial t^{2}}+\gamma \frac{\partial w}{\partial t}+\frac{\partial^{2} M_{i n t}}{\partial x^{2}}=g(t, x)+\frac{\partial^{2} M_{p z t}}{\partial x^{2}} \\
& w(t, 0)=\frac{\partial w}{\partial x}(t, 0)=0 \\
& \mathcal{M}_{\text {int }}(t, \ell)=\frac{\partial \mathcal{M}_{i n t}}{\partial x}(t, \ell)=0
\end{aligned}
$$

where the composite density is

$$
\rho(x)= \begin{cases}\rho_{b} h_{b} b+2 \rho_{p z t} h_{p z t} b & , \quad p_{1} \leq x \leq p_{2} \\ \rho_{b} h_{b} b & , \text { otherwise }\end{cases}
$$

The internal bending moment is

$$
M_{i n t}(t, x)=E I(x) \frac{\partial^{2} w}{\partial x^{2}}+c_{D} I(x) \frac{\partial^{3} w}{\partial x^{2} d t}
$$

where the spatially varying stiffness and damping parameters are defined by

$$
\begin{gathered}
E I(x)= \begin{cases}\frac{E_{b} h_{b}^{3} b}{12}+\frac{2 b}{3} E_{p z t} a_{3} & , \quad p_{1} \leq x \leq p_{2} \\
\frac{E_{b} h_{b}^{3} b}{12} & , \text { otherwise }\end{cases} \\
c_{D} I(x)= \begin{cases}\frac{c_{D} h_{b}^{3} b}{12}+\frac{2 b}{3} c_{p z t} a_{3} & , \quad p_{1} \leq x \leq p_{2} \\
\frac{c_{D} h_{b}^{3} b}{12} & , \text { otherwise }\end{cases}
\end{gathered}
$$

where $a_{3}=\left(h_{b} / 2+h_{p z t}\right)^{3}-h_{b}^{3} / 8$ (details regarding the formulation of these parameters can be found in $[3,12])$. In applications, the piecewise constant parameters $\rho(x), E I(x)$ and $c_{D} I(x)$ are typically estimated through a least squares fit to data.

Under the assumption of linear piezoceramic responses, the external bending moment generated through the application of diametrically out-of-phase voltages $u(t)$ to the patches is given by

$$
M_{e x t}(t, x)=E_{p z t} d_{31}\left(h_{b}+h_{p z t}\right) u(t) \chi_{p z t}(x) .
$$

Here $d_{31}$ denotes the piezoelectric coupling coefficient and the characteristic function

$$
\chi_{p z t}(x)= \begin{cases}1 & , \quad p_{1} \leq x \leq p_{2} \\ 0 & , \quad \text { otherwise }\end{cases}
$$

isolates inputs to the region covered by the patches. We note that the relation (3) is valid only at low to moderate drive levels when constitutive nonlinearities and hysteresis are minimal. For higher 
drive levels, nonlinear hysteresis models of the type discussed in $[6,8,10,13,14]$ must be employed when quantifying external moments.

The strong form of the model (1) requires the derivatives of discontinuous material parameters and patch coefficients with the latter yielding highly unbounded control inputs (derivatives of the Dirac distribution). Furthermore, numerical approximation of the strong form of the model would require the use of high-order basis functions for Galerkin methods due to the fourth-order spatial derivatives. To provide a formulation which is amenable to approximation and subsequent finite-dimensional control design, it is advantageous to consider a weak formulation of the model in which the state space is $X=L^{2}(0, \ell)$ and the space of test functions is taken to be $V=\left\{\phi \in H^{2}(0, \ell) \mid \phi(0)=\phi^{\prime}(0)=0\right\}$. As detailed in [3], either integration by parts or the application of Hamiltonian energy principles then yields the corresponding weak form of the model

$$
\begin{gathered}
\int_{0}^{\ell} \rho(x) \ddot{y} \phi d x+\gamma \int_{0}^{\ell} \dot{w} \phi d x+\int_{0}^{\ell} E I(x) w^{\prime \prime} \phi^{\prime \prime} d x+\int_{0}^{\ell} c_{D} I(x) \dot{w}^{\prime \prime} \phi^{\prime \prime} d x \\
=\int_{0}^{\ell} g(t, x) \phi d x+\int_{0}^{\ell} M_{p z t} \phi^{\prime \prime} d x
\end{gathered}
$$

which must be satisfied for all $\phi \in V$.

To obtain a corresponding semi-discrete system appropriate for control implementation, we employ Galerkin approximations in space with temporally-varying coefficients. To satisfy smoothness requirements while minimizing system size, the basis is comprised of cubic B-splines modified to satisfy the essential boundary conditions. For a uniform partition of $[0, \ell]$ with gridpoints $x_{j}=j h, h=\ell / N, j=0, \cdots, N$, the basis functions $\left\{\phi_{j}\right\}_{j=1}^{N+1}$ are defined by

$$
\begin{aligned}
& \phi_{1}(x)=-2 s_{-1}(x)+s_{0}(x)-2 s_{1}(x) \\
& \phi_{j}(x)=s_{j}(x) \quad, \quad j=2, \cdots, N+1
\end{aligned}
$$

where

$$
s_{j}(x)=\frac{1}{h^{3}}\left\{\begin{array}{lll}
\left(x_{j+2}-x\right)^{3} & , \quad x_{j+1}<x \leq x_{j+2} \\
-3\left(x_{j+1}-x\right)^{3}+3 h\left(x_{j+1}-x\right)^{2}+3 h^{2}\left(x_{j+1}-x\right)+h^{3} & , & x_{j}<x \leq x_{j+1} \\
-3\left(x-x_{j-1}\right)^{3}+3 h\left(x-x_{j-1}\right)^{2}+3 h^{2}\left(x-x_{j-1}\right)+h^{3} & , & x_{j-1}<x \leq x_{j} \\
\left(x-x_{j-2}\right)^{3} & , & x_{j-2}<x \leq x_{j-1} \\
0 & , & \text { otherwise }
\end{array}\right.
$$

(see [9]). It should be noted that with this choice of basis, $H^{N}=\operatorname{span}\left\{\phi_{i}\right\} \subset V$. Approximate solutions are then specified as

$$
w^{N}(t, x)=\sum_{j=1}^{N+1} w_{j}(t) \phi_{j}(x) .
$$

To obtain a finite dimensional system, the weak form of the model is projected into the finite dimensional space $H^{N}$ to obtain

$$
\begin{gathered}
\sum_{j=1}^{N+1} \ddot{w}_{j} \int_{0}^{\ell} \rho(x) \phi_{j} \phi_{i} d x+\sum_{j=1}^{N+1} \dot{w}_{j} \gamma \int_{0}^{\ell} \phi_{j} \phi_{i} d x+\sum_{j=1}^{N+1} w_{j} \int_{0}^{\ell} E I(x) \phi_{j}^{\prime \prime} \phi_{i}^{\prime \prime} d x \\
+\sum_{j=1}^{N+1} \dot{w}_{j} \int_{0}^{\ell} c_{D} I(x) \phi_{j}^{\prime \prime} \phi_{i}^{\prime \prime} d x=\int_{0}^{\ell} g(t, x) \phi_{i} d x+\int_{0}^{\ell} M_{p z t} \phi_{i}^{\prime \prime} d x
\end{gathered}
$$


for all $\phi_{i} \in H^{N}$. Employing matrix notation, the second-order system can be formulated as

$$
M \ddot{\vec{w}}+C \dot{\vec{w}}+K \vec{w}=\vec{b} u(t)+\vec{f}
$$

where $\vec{w}(t)=\left[w_{1}(t), \cdots, w_{N+1}(t)\right]$. The mass, damping and stiffness matrices, along with the force and control input vectors, have the components

$$
\begin{aligned}
& {[M]_{i j}=\int_{0}^{\ell} \rho(x) \phi_{i} \phi_{j} d x} \\
& {[C]_{i j}=\gamma \int_{0}^{\ell} \phi_{i} \phi_{j} d x+\int_{0}^{\ell} c_{D} I(x) \phi_{j}^{\prime \prime} \phi_{i}^{\prime \prime} d x} \\
& {[K]_{i j}=\int_{0}^{\ell} E I(x) \phi_{i}^{\prime \prime} \phi_{j}^{\prime \prime} d x} \\
& {[\vec{f}]_{i}=\int_{0}^{\ell} g(t, x) \phi_{i} d x} \\
& {[\vec{b}]_{i}=K_{b} \int_{p_{1}}^{p_{2}} \phi_{i}^{\prime \prime} d x}
\end{aligned}
$$

where $K_{b}=E_{p z t} d_{31}\left(h_{b}+h_{p e}\right)$. Letting $y(t)=[\vec{w}(t), \dot{\vec{w}}(t)]^{T}$ and

$$
A=\left[\begin{array}{cc}
0 & I \\
-M^{-1} K & -M^{-1} C
\end{array}\right] \quad, \quad F(t)=\left[\begin{array}{c}
0 \\
M^{-1} \vec{f}(t)
\end{array}\right] \quad, \quad B(t)=\left[\begin{array}{c}
0 \\
M^{-1} \vec{b}(t)
\end{array}\right],
$$

the second-order system (7) can be posed as the first-order system

$$
\begin{aligned}
& \dot{y}(t)=A y(t)+B u(t)+F(t) \\
& \vec{y}(0)=\vec{y}_{0},
\end{aligned}
$$

where the $2 N \times 1$ vector $\vec{y}_{0}$ denotes the projection of the initial conditions into $H^{N}$. The finite dimensional model (9) can then be used for subsequent control design.

We point out that for more complex physical systems, modal or finite element techniques can be employed to construct finite dimensional models of the form defined in (9). Once such models are constructed, the control techniques described in the next section can be employed.

\section{Control Design}

There exist a large number of classical, adaptive, optimal, and robust control techniques which can be employed to specify controls $u$ for the system (9). To achieve the goal of ultimately determining optimal capabilities of certain smart structural designs, we focus here on certain optimal control techniques. In addition to determining design capabilities, an important criterion is to develop methods which are sufficiently efficient and robust to permit real-time implementation.

Even within the realm of optimal control design, there exist a number of feasible approaches. We summarize here two classical formulations based on time domain cost functionals and then consider a narrowband design based on frequency domain criteria. The first two approaches are well documented in the literature for smart material control design and we summarize here only those details which are pertinent for comparison with the narrowband design. 
We consider first the case in which the exogenous force $F$ in (9) is comprised of a linear combination of temporally periodic functions with common period $\tau$. We then consider the minimization of the quadratic functional

$$
J(u)=\frac{1}{2} \int_{0}^{\tau}\{\langle Q y, y\rangle+\langle R u, u\rangle\} d t
$$

subject to

$$
\begin{aligned}
& \dot{y}(t)=A y(t)+B u(t)+F(t) \\
& y(0)=y(\tau) .
\end{aligned}
$$

Here $\langle\cdot, \cdot\rangle$ denotes the Euclidean inner product in $\mathbb{R}^{N+1}$. The matrix $Q$ which weights the states is often taken to be

$$
Q=\left[\begin{array}{cc}
d_{1} K & 0 \\
0 & d_{2} M
\end{array}\right]
$$

where $M$ and $K$ are the mass and stiffness matrices defined in (8) since this is the identity with regard to the product space inner product. The control weight $R$ is typically a scalar, or in the case of multiple inputs, a scalar multiple of an identity matrix having the correct dimensions. Further details regarding the construction of both matrices can be found in [1]. As detailed in [3, Chapters 8 and 9], or [5] for the infinite dimensional problem, the optimal control which minimizes (10) is given in feedback form by

$$
u(t)=-R^{-1} B^{T}[\Pi z(t)-r(t)]
$$

where the $\tau$-periodic trajectory $z(t)$ is obtained by solving the closed loop system

$$
\begin{aligned}
& \dot{z}(t)=\left[A-B R^{-1} B^{T} \Pi\right] z(t)-B R^{-1} B^{T} r(t)+F(t) \\
& z(0)=z(\tau) .
\end{aligned}
$$

The Riccati variable $\Pi$ and tracking variable $r(t)$ respectively satisfy the equations

$$
A^{T} \Pi+\Pi A-\Pi B R^{-1} B^{T} \Pi+Q=0
$$

and

$$
\begin{aligned}
& \dot{r}(t)=-\left[A-\Pi B R^{-1} B^{T}\right]^{T} r(t)+\Pi F(t) \\
& r(0)=r(\tau) .
\end{aligned}
$$

In practice, the solution of the boundary value problem (15) is typically simplified by considering the final time condition $r(\tau)=0$ and time-marching the solution forward in negative time in lieu of enforcing the boundary condition $r(0)=r(\tau)$. In spite of this approximation, however, the determination of an optimal control $u$ is costly since it requires the solution, storage, and possible updating of tracking trajectories $r(t)$. Furthermore, the determination of $r$ is often performed prior to closing the loop which produces robustness issues due to potentially unincorporated phase shifts. Hence, while the control law (12) has been experimentally implemented [2], the robustness issues and complexity associated with the determination of $r(t)$ render this approach more feasible as a theoretical or numerical design tool than an algorithm to be implemented in physical systems.

A second approach is to design a feedback law for the unforced system to obtain suboptimal attenuation in the presence of an exogenous force. This is accomplished by minimizing the functional

$$
J(u)=\frac{1}{2} \int_{0}^{\infty}\{\langle Q y, y\rangle+\langle R u, u\rangle\} d t
$$


subject to

$$
\begin{aligned}
& \dot{y}(t)=A y(t)+B u(t) \\
& y(0)=y_{0} .
\end{aligned}
$$

The control in this case is

$$
u(t)=-R^{-1} B^{T} \Pi y(t)
$$

where $\Pi$ again solves the algebraic Riccati equation (ARE) (14). The use of the feedback law (18) in the original system (9) will attenuate stochastic disturbances or model uncertainties but will have less effect on harmonic disturbances or steady state system dynamics due to periodic exogenous forces.

To develop a feedback law which targets either natural frequencies for the system, or specific frequencies in the exogenous force, it is advantageous to consider a frequency-domain functional of the form

$$
J(u)=\frac{1}{2} \int_{0}^{\infty}\left[y^{*}(i \omega) Q(i \omega) y(i \omega)+u^{*}(i \omega) R u(i \omega)\right] d \omega
$$

where ${ }^{*}$ represents the complex conjugate transpose and $Q(i \omega), R(i \omega)$ again denote design parameters. In order to transform this frequency domain cost functional into an equivalent time domain cost functional, consider the input/output relation given by the transfer function $P(s)$

$$
\widetilde{y}(s)=P(s) y(s) .
$$

If $P(s)$ is a ratio of polynomials and the number of zeros of $P$ does not exceed the number of poles, then (20) can be expressed as the linear system

$$
\begin{aligned}
& \dot{z}=\widetilde{A} z+\widetilde{B} y \\
& \tilde{v}=\widetilde{C} z+\widetilde{D} y .
\end{aligned}
$$

To determine appropriate choices for $\widetilde{A}, \widetilde{B}, \widetilde{C}, \widetilde{D}$, we note that minimization of the alternative frequency domain cost functional

$$
\begin{aligned}
J(u) & =\frac{1}{2} \int_{0}^{\infty}\left[\tilde{y}^{*}(i \omega) \tilde{y}(i \omega)+u^{*}(i \omega) R u(i \omega)\right] d \omega \\
& =\frac{1}{2} \int_{0}^{\infty}\left[y^{*}(i \omega) P^{*}(i \omega) P(i \omega) y(i \omega)+u^{*}(i \omega) R u(i \omega)\right] d \omega
\end{aligned}
$$

is equivalent to minimizing (19) when $Q$ is defined by

$$
Q(i \omega)=P^{*}(i \omega) P(i \omega) .
$$

Defining $Q(i \omega)$ in this manner satisfies the necessary condition that equation (22) yields a nonnegative real number. The choice of $\mathrm{P}$ used here is

$$
P(i \omega)=\frac{\omega_{r}^{2} i \omega}{\omega_{r}^{2}-\omega^{2}+2 \omega_{r} \xi i \omega}
$$

where $\xi$ is a design parameter and $\omega_{r}$ is the lowest known resonant frequency [7, 11]. Note that $Q(i \omega)$ penalizes system responses to frequencies in a neighborhood of the lowest resonant frequency. The size of the frequency range is dictated by the design parameter $\xi$. Figure 2 demonstrates the behavior of $Q(i \omega)$ for three values of $\xi$ and a resonant frequency of $\omega_{r}=35$ radians. 


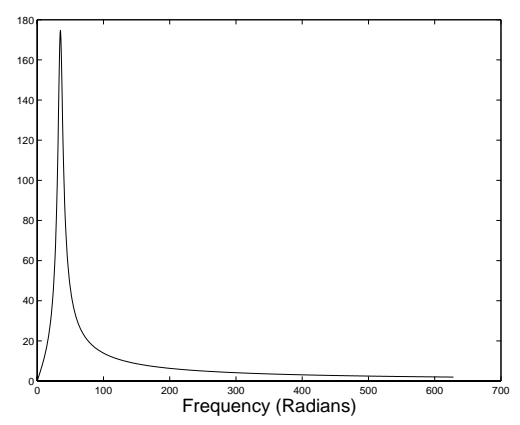

(a)

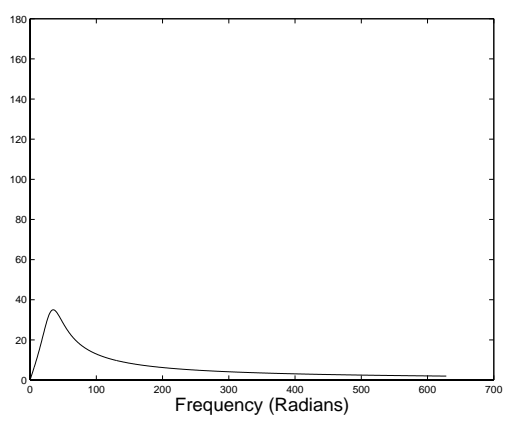

(b)

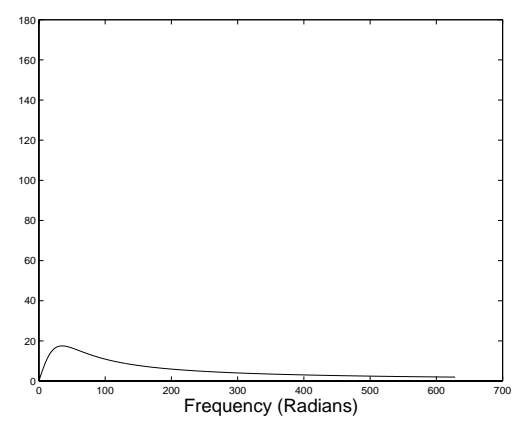

(c)

Figure 2. Profiles of $Q(i \omega)$ for (a) $\xi=0.1$; (b) $\xi=0.5$ and (c) $\xi=1$.

From (20) and (21) it follows that an associated differential equation system for the choice (23) for $P$ is

$$
\left[\begin{array}{c}
z_{1}^{r} \\
z_{2}^{r}
\end{array}\right]=\left[\begin{array}{cc}
0 & 1 \\
-\omega_{r}^{2} & -2 \omega_{r} \xi
\end{array}\right]\left[\begin{array}{c}
z_{1}^{r} \\
z_{2}^{r}
\end{array}\right]+\left[\begin{array}{c}
0 \\
\widetilde{B}_{1}
\end{array}\right] v(t)
$$

where $\widetilde{B}_{1}$ is a row matrix that weights the elements of $v$.

As illustrated in [7], minimization of (19) is equivalent to minimizing the cost functional associated with the augmented system

$$
\left[\begin{array}{c}
y \\
z_{1}^{r} \\
z_{2}^{r}
\end{array}\right]=\left[\begin{array}{ccc}
A & 0 & 0 \\
0 & 0 & 1 \\
\widetilde{B}_{1} & -\omega_{r}^{2} & -2 \omega_{r} \xi
\end{array}\right]\left[\begin{array}{c}
y \\
z_{1}^{r} \\
z_{2}^{r}
\end{array}\right]+\left[\begin{array}{c}
B \\
0 \\
0
\end{array}\right] u(t)+\left[\begin{array}{c}
F(t) \\
0 \\
0
\end{array}\right]
$$

If the augmented state is denoted by $y_{1}=\left[y^{T}, z_{1}^{r}, z_{2}^{r}\right]^{T},(25)$ can be formulated as

$$
\dot{y}_{1}=A_{1} y_{1}+B_{1} u(t)+F_{1}(t)
$$

where the definitions of $A_{1}, B_{1}$ and $F_{1}(t)$ follow from (25). By considering the system (26), LQR techniques can be employed to compute frequency-shaped Riccati solutions $\Pi$ and corresponding feedback gains.

In a similar manner, the system response to two frequencies $\omega_{r}$ and $\omega_{d}$ can be minimized through consideration of the augmented system

$$
\left[\begin{array}{c}
y \\
z_{1}^{r} \\
z_{2}^{r} \\
z_{1}^{d} \\
z_{2}^{d}
\end{array}\right]=\left[\begin{array}{ccccc}
A & 0 & 0 & 0 & 0 \\
0 & 0 & 1 & 0 & 0 \\
\widetilde{B}_{1} & -\omega_{r}^{2} & -2 \omega_{r} \xi_{r} & 0 & 0 \\
0 & 0 & 0 & 0 & 1 \\
\widetilde{B}_{2} & 0 & 0 & -\omega_{d}^{2} & -2 \omega_{d} \xi_{d}
\end{array}\right]\left[\begin{array}{c}
y \\
z_{1}^{r} \\
z_{2}^{r} \\
z_{1}^{d} \\
z_{2}^{d}
\end{array}\right]+\left[\begin{array}{c}
B \\
0 \\
0 \\
0 \\
0
\end{array}\right] u(t)+\left[\begin{array}{c}
F(t) \\
0 \\
0 \\
0 \\
0
\end{array}\right]
$$

where $\widetilde{B}_{2}$ is a second design parameter. For $y_{2}=\left[y^{T}, z_{1}^{r}, z_{2}^{r}, z_{1}^{d}, z_{2}^{d}\right]^{T}$, the system can be posed as

$$
\dot{y}_{2}=A_{2} y_{2}+B_{2} u(t)+F_{2}(t) \text {. }
$$

We note that additional frequencies can be targeted in a similar manner. 
Thus through the consideration of a frequency domain functional and associated augmented systems in the time domain, one obtains a feedback formulation that is no more complex to implement than standard LQR laws but targets specified system or exogenous frequencies. This significantly enhances the feasibility of experimentally implementing the method in physical systems while achieving the control authority required when employing PZT actuators in smart structures. Numerical examples illustrating attributes of this narrowband control approach are provided in the next section.

Finally, we note that this narrowband design can be combined with standard LQR feedback techniques to augment the effectiveness of the controller for attenuating unmodeled dynamics or stochastic disturbances. The development of such hybrid control approaches has been illustrated in [4] in the context of narrowband adaptive techniques and similar hybrid techniques are currently being developed which utilize the narrowband controls discussed here.

\section{Numerical Examples}

To illustrate the performance of the narrowband control method, we consider the response of the prototypical system to a $600 \mathrm{~Hz}$ exogenous force; hence $g(t, x)=\sin (2 \pi \cdot 600 t)$ in (4) and the corresponding finite dimensional model. The length of the beam was taken to be $\ell=.4573 \mathrm{~m}$ and the coordinates for the patch pair were $p_{1}=0.2 \mathrm{~m}$ and $p_{2}=0.25 \mathrm{~m}$. The density, stiffness, damping and control input parameters were taken to be $\rho_{b}=0.093 \mathrm{~kg} / \mathrm{m}, \rho_{p z t}=0.443 \mathrm{~kg} / \mathrm{m}, E I_{b}=0.491 \mathrm{Nm}^{2}$, $E I_{p z t}=0.793 \mathrm{Nm}^{2}, c_{D}=6.49 \times 10^{-6} \mathrm{sNm}^{2}, c_{p z t}=1.225 \times 10^{-5} \mathrm{sNm}^{2}, \gamma=0.013 \mathrm{sN} / \mathrm{m}^{2}$ and $K_{b}=1.746 \times 10^{-2} \mathrm{Nm} / \mathrm{V}$. For this set of dimensions and parameter choices, the beam has a primary resonant frequency of $5.5 \mathrm{~Hz}$ and a secondary resonant frequency of $32 \mathrm{~Hz}$.

The uncontrolled displacement at the point $x=3 \ell / 5$ and the closed loop displacement obtained with the LQR law (18) are compared in Figure 3a while the corresponding velocities are plotted in Figure $3 \mathrm{~b}$. The control was initiated at 0.74 seconds and for the presented simulations, the design parameters were specified as

$$
Q=5\left[\begin{array}{cc}
K & 0 \\
0 & M
\end{array}\right] \quad, \quad R=1 \times 10^{-4}
$$

It is observed that while the general feedback law reduces the displacements by an order of magnitude within 1.5 seconds of control implementation, it did not reduce the high frequency $600 \mathrm{~Hz}$ component reflected in the velocity. This is further illustrated in the FFT of the beam response from 1.25 to 2.5 seconds which is plotted in Figure 3c. This latter figure illustrates that the $600 \mathrm{~Hz}$ component of the beam response is actually unaffected by the feedback law (18). This is to be expected since this formulation does not incorporate any information about the exogenous force into the penalty functional or feedback law.

We next illustrate the performance of the narrowband controller which is designed to attenuate either specific natural or exogenous frequencies. We consider first a penalty functional that targets the $5.5 \mathrm{~Hz}$ primary frequency. The parameters in the augmented system (26) were taken to be $\omega_{r}=5.5(2 \pi)$ and $\xi=1$. The design parameters for the augmented system were specified as

$$
Q_{1}=\left[\begin{array}{ll}
Q & 0 \\
0 & I
\end{array}\right] \quad, \quad R_{1}=0.001
$$

where $Q$ is specified in (29).

The displacement obtained in this case is compared with that resulting from the LQR law (18) in Figure 4. It is observed that the frequency-shaped response exhibits nearly critical damping of 


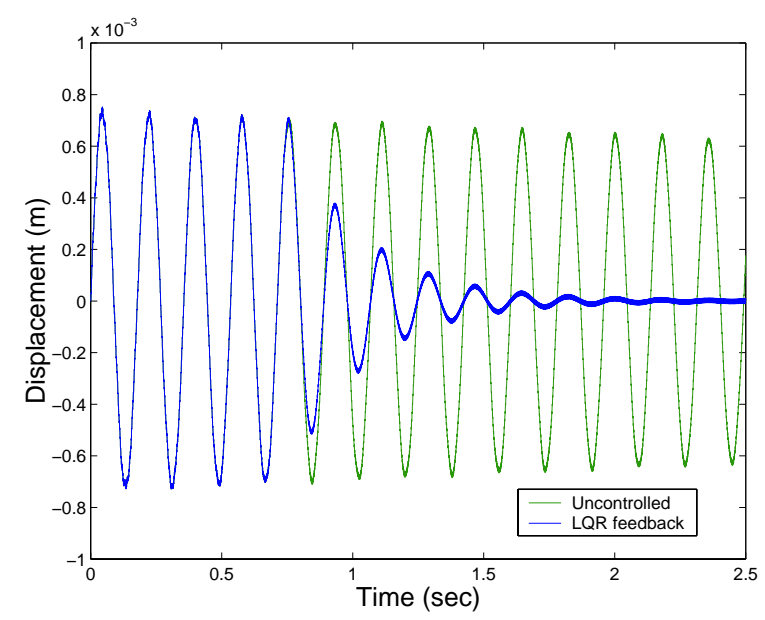

(a)

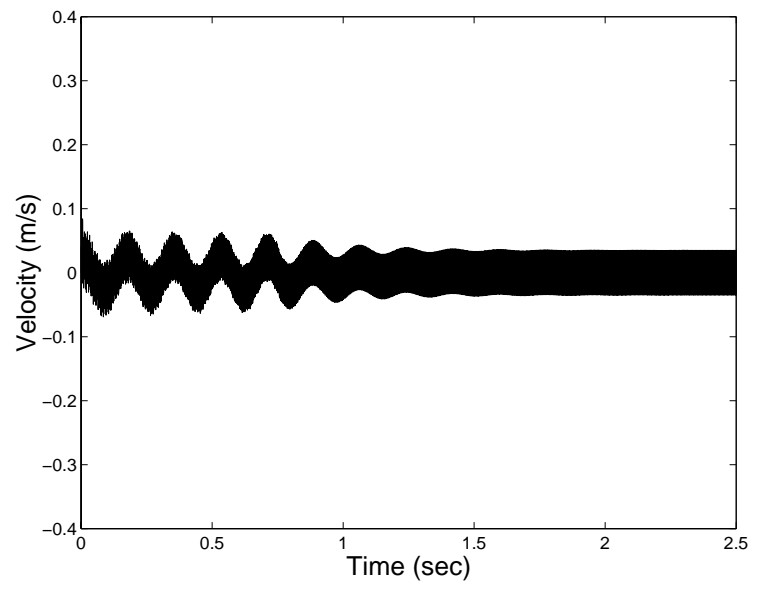

(b)
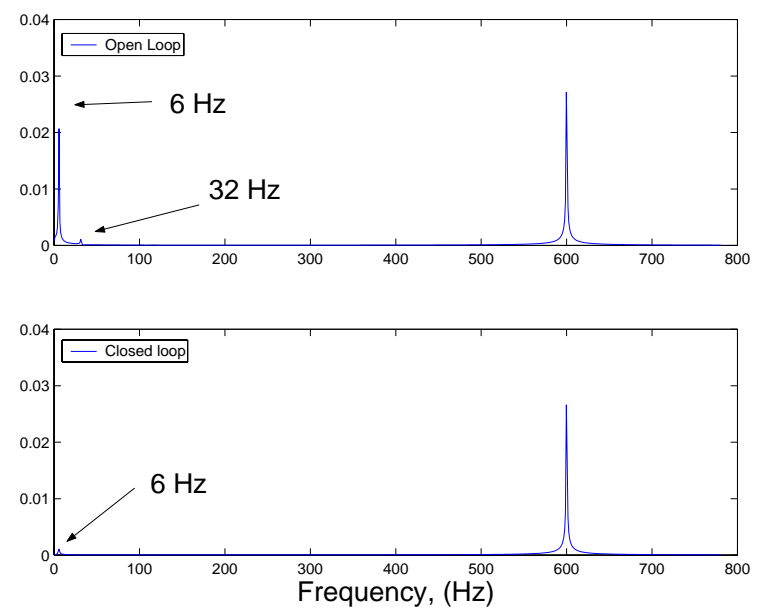

(c)

Figure 3. (a) Open and closed loop displacements obtained with the feedback law (18); (b) Open and closed loop velocities; (c) FFT of velocities over the time interval [1.25, 2.5].

the primary $5.5 \mathrm{~Hz}$ response but there is essentially no attenuation of the steady state response due to the exogenous force since this information is not included in the control formulation.

To minimize both the transient response and the $600 \mathrm{~Hz}$ steady state response, we subsequently employed the formulation (28) with $\omega_{r}=5.5$ and $\omega_{d}=600$ is employed along with the design parameters $R=0.001$ and

$$
Q_{2}=\left[\begin{array}{ccc}
Q & 0 & 0 \\
0 & 10 I & 0 \\
0 & 0 & I
\end{array}\right]
$$

The resulting displacement and velocity are plotted in Figure 5. It is observed that by 1.5 seconds, both states are effectively attenuated. This is reiterated in the FFT of the displacement (not shown here) which indicates negligible energy at $600 \mathrm{~Hz}$ after 1.5 seconds. This provides initial verification regarding the effectiveness of the narrowband control design. 


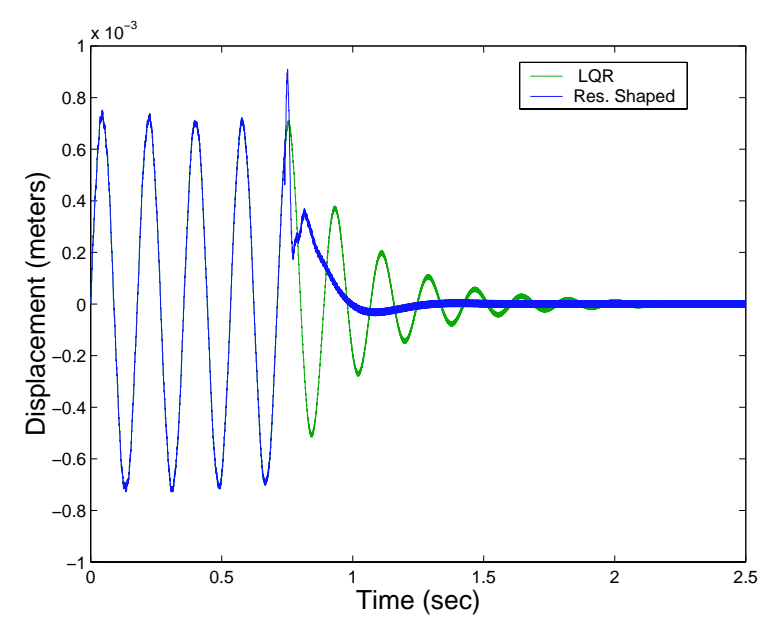

(a)

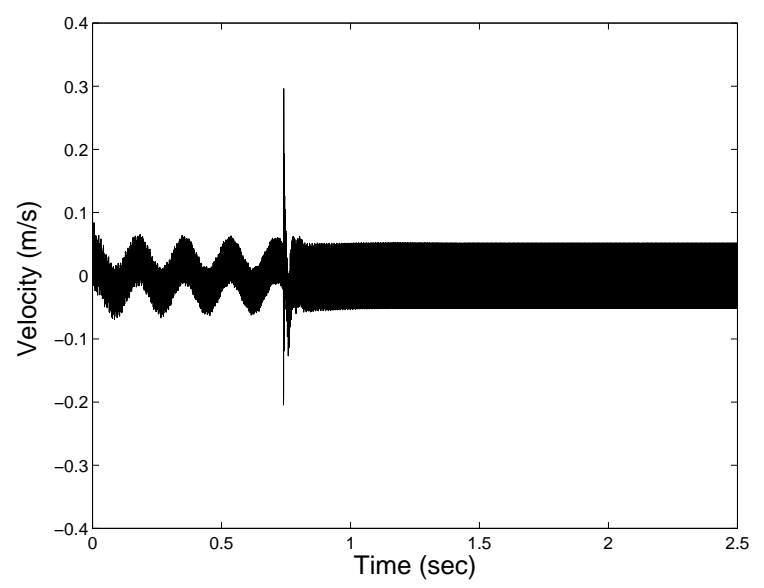

(b)

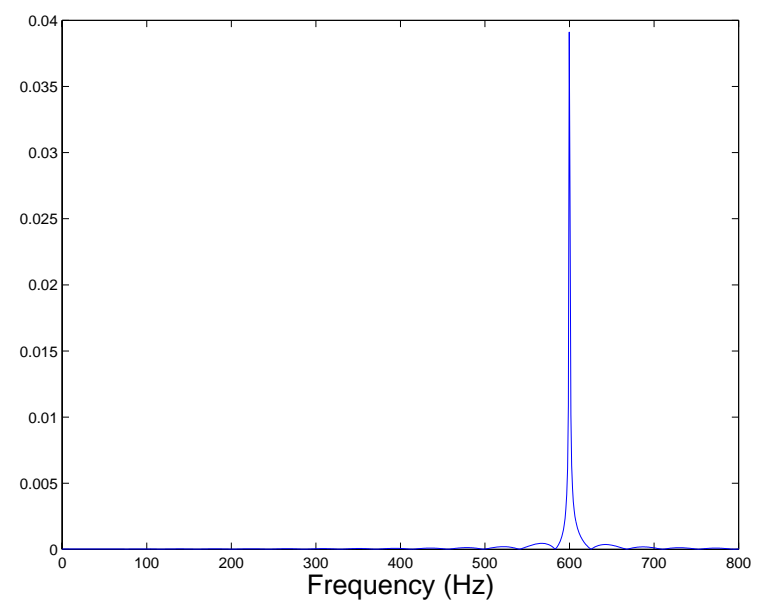

(c)

Figure 4. (a) Closed loop displacement obtained with the feedback law (18) and LQR design for the augmented system (26) employing $\omega_{r}=5.5$; (b) Closed loop velocities; (c) Fft of velocities over the time interval $[1.25,2.5]$.

\section{Concluding Remarks}

A narrowband optimal control methodology for smart structures has been outlined and illustrated for a prototypical structure with piezoceramic actuators. The criteria for this method were twofold: (i) to provide effective attenuation in the presence of exogenous disturbances and (ii) to be sufficiently efficient and robust to permit real-time implementation in physical systems. The first criterion can be achieved through the formulation of feedback laws which include tracking variables; however, the complexity of these formulations leads to robustness issues and often precludes direct implementation.

The control approach considered here employs a frequency domain functional to target specified frequencies. For implementation purposes, a corresponding augmented system is formed which contains the original linear model as a subsystem. Because standard LQR techniques can be employed to compute gains for the augmented system (which in turn contains the necessary information for frequency targeting), the method is as efficient to implement as standard LQR feedback techniques. 


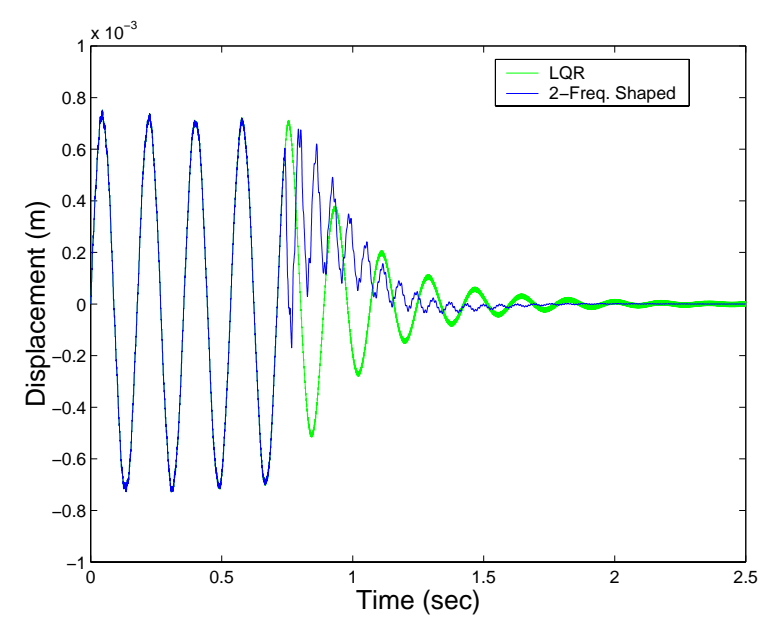

(a)

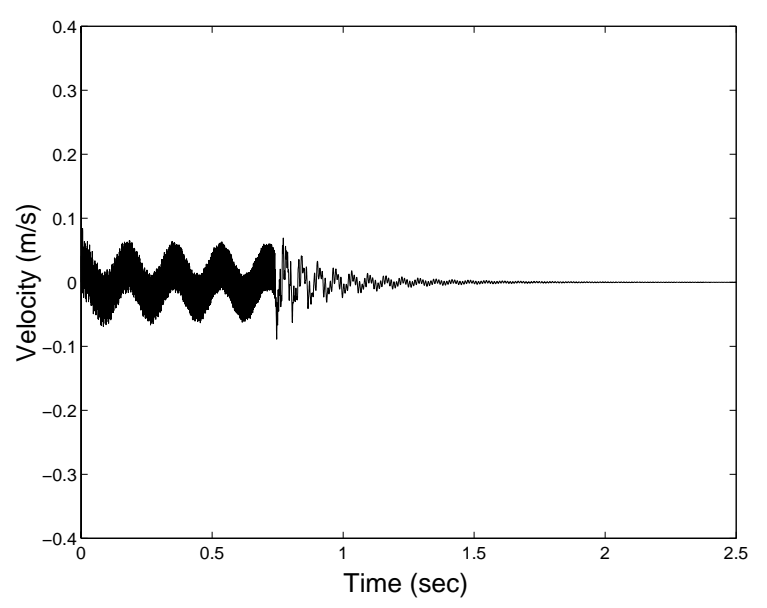

(b)

Figure 5. (a) Closed loop displacement obtained with the feedback law (18) and LQR design for the augmented system (28) employing $\omega_{r}=5.5$ and $\omega_{d}=600$; (b) Closed loop velocities.

Initial verification regarding the validity of the method is provided by numerical examples. Current investigations are focused on the experimental implementation of the method to ascertain its effectiveness for physical systems.

\section{Acknowledgements}

This research was supported in part by the Air Force Office of Scientific Research under the grant AFOSR-F49620-01-1-0107.

\section{References}

[1] H.T. Banks, W. Fang, R.J. Silcox and R.C. Smith, "Approximation methods for control of acoustic/structure models with piezoceramic actuators," Journal of Intelligent Material Systems and Structures, 4(1), 1993, pp. 98-116.

[2] H.T. Banks, R.C. Smith, D.E. Brown, R.J. Silcox and V.L. Metcalf, "Experimental Confirmation of a PDE-Based Approach to Design of Feedback Controls," SIAM Journal on Control and Optimization, 35(4), 1997, pp. 1263-1296.

[3] H.T. Banks, R.C. Smith and Y. Wang, Smart Material Structures: Modeling, Estimation and Control, Masson/John Wiley, Paris/Chichester, 1996.

[4] R.L. Clark, W.R. Saunders and G.P. Gibbs, Adaptive Structures: Dynamics and Control, John Wiley \& Sons, Inc., New York, 1998.

[5] G. Da Prato, "Synthesis of optimal control for an infinite dimensional periodic problem," SIAM Journal of Control and Optimization, 25(3), pp. 706-714, 1987.

[6] P. Ge and M. Jouaneh, "Generalized Preisach model for hysteresis nonlinearity of piezoceramic actuators," Precision Engineering, 20, pp. 99-111, 1997. 
[7] N.K. Gupta, "Frequency-shaped cost functionals: extension of linear quadratic Gaussian design methods," Journal of Guidance and Control, 3(6), 1980.

[8] D. Hughes and J.T. Wen, "Preisach modeling of piezoceramic and shape memory alloy hysteresis," Smart Materials and Structures, 6, pp. 287-300, 1997.

[9] P.M. Prenter, Splines and Variational Methods, Wiley Classics Edition, New York, 1989.

[10] T.J. Royston and B.H. Houston, "Modeling and measurement of nonlinear dynamic behavior in piezoelectric ceramics with application to 1-3 composites," Journal of the Acoustical Society of America, 104, pp. 2814-2827, 1998.

[11] L.A. Sievers and A.H. von Flotow, "Comparison of two LQG-based methods for disturbance rejection," Proceedings of the 28th IEEE Conference on Decision and Control, Tampa, FL, Dec. 1989.

[12] R.C. Smith, "A nonlinear optimal control method for magnetostrictive actuators," Journal of Intelligent Material Systems and Structures, 9(6), 1998, pp. 468-486.

[13] R.C. Smith and Z. Ounaies, "A domain wall model for hysteresis in piezoelectric materials," Journal of Intelligent Material Systems and Structures, 11(1), 2000, pp. 62-79.

[14] R.C. Smith, Z. Ounaies and R. Wieman, "A model for rate-dependent hysteresis in piezoceramic materials operating at low frequencies," Proceedings of the SPIE, Smart Structures and Materials 2000, Newport Beach, CA, Volume 3992, pp. 128-136, 2000. 